\title{
SPACE-CHARGE EFFECTS ON THE BEAM RESONANCE INSTABILITY
}

\author{
M. B. Ottinger, and T. Tajima,University of Texas, Austin, TX 78703 and \\ K. Hiramoto, Hitachi Research Lab, Hitachi Ltd. Hitachi-shi, Ibaraki-ken, 316, Japan
}

\section{Abstract}

We study the space-charge effects on the stability of low energy synchrotron accelerators with a Gaussian beam distribution. We show that the tune shift is not uniform throughout the beam, but decreases as a function of particle amplitude. The amplitude dependance leads to an equation for the region of beam instability due to integer resonance.

\section{INTRODUCTION}

One of the major concerns of beam dynamics and thus beam quality for a synchrotron in low energies (such as low energy injector/booster and medical proton therapy) is the space charge effect. In the nonrelativistic, low energy regime the repulsive space charge electric force is not cancelled out by the magnetic force, thus yielding a substantial modification in beam dynamics, such as the tune shift [1]. In fact, it has been believed that the space charge effect increases the emittance. A rule of thumb on how to avoid or contain this deliterious effect is to avoid any conditions where the shifted tune might overlap integer (or other rational) resonances. Experimental observations, however, show that in many cases even though the beam has evidently crossed such a resonance, the beam is not destroyed. This has remained a mystery and some attempts at understanding the effects have been tried $[2,3,4]$ but to no clear understanding to date. In this paper we address this problem by looking into the detailed kinetic radial distribution with the employment of our newly developed self-consistent space-charge PIC code [5].

In their 1958 paper on the theory of the alternating gradient synchrotron[6] E.D. Courant and H.S. Snyder show that magnet imperfections, and other non-linear effects, produce two classes of instabilities. The first occurs when either transverse beam tune (number of transverse oscillations the beam particles make around the design orbit per synchrotron revolution) is equal to a rational number $\left(\nu_{\mathrm{o}}=\mathrm{n} / \mathrm{m}\right.$, where $\mathrm{n}$ and $\mathrm{m}$ are integers). The second class of instabilities occur when the sum of multiples of the transverse tunes is equal to an integer value $\left(n v_{x o}+m v_{y o}=p\right)$. In their paper, Courant and Snyder used the machine tunes, or the number of transverse oscillations produced by a particle's interaction with only the synchrotron magnets. For low energy/high intensity beams the particle trajectory will also be influenced by the beam's self-fields, or spacecharge. For a uniform density beam the space-charge produces a uniform shift in the beam tune. R.C.
Davidson[7] calculates the average tune in a circular, uniform density beam with space-charge as

$$
v=\left[v_{o}^{2}+\left(\frac{K S}{2 \varepsilon}\right)^{2}\right]^{\frac{1}{2}}-\frac{K S}{2 \varepsilon},
$$

where $S$ is the machine circumference, $\varepsilon$ is the unnormalized beam emittance, and $\mathrm{K}$ is the self-field perveance $\left(K=2 \operatorname{Iem}^{-1}[\gamma \beta c]^{-3}\right)$.

For small K, the tune can be written in terms of the machine tune and a space-charge tune-shift,

$$
v=v_{o}-\Delta \bar{v}, \quad \text { where } \Delta \bar{v}=\frac{1}{2} K S \varepsilon^{-1} \text {. }
$$

Eqs. (1) and (2) were derived for a uniform density beam. To expand it to a Gaussian beam, the emittance is generally replaced by the rms emittance. Since the space-charge has been shown to produce a uniform shift in tune, it has been preported that the instabilities shown by Courant and Snyder occur at the new shifted tunes

$$
\begin{gathered}
v_{o}-\Delta \bar{v}=\frac{n}{m} \\
n\left(v_{x o}-\Delta \overline{v_{x}}\right)+m\left(v_{y o}-\Delta \overline{v_{y}}\right)=p .
\end{gathered}
$$

Baartman refutes this notion in his paper[3]. He proposes that the resonance is associated with the incoherent tune, which can be depressed beyond the integral value by a fractional amount determined by the machine design. The resonance occurs at

$$
v_{o}-C_{m k} \Delta \bar{v}=n / m \text {, }
$$

where $\mathrm{C}_{\mathrm{mk}}$ is a function of the machine design.

In each of the prior studies the tune-shift is considered uniform over the beam. W.T. Weng[4] briefly shows that this is not the case, the tune-shift, for a non-uniform beam is dependent upon the individual particle amplitude.

\section{AMPLITUDE DEPENDENCE OF TUNE SHIFT}

Consider a long, circular proton beam with a Gaussian radial density profile, $\rho(r)=\rho_{o} e^{-\frac{r^{2}}{a^{2}}}$. From Maxwell's equations the electric and magnetic fields are:

$$
\begin{gathered}
\vec{E}(r)=E_{r}(r)=\frac{a^{2} \rho_{o}}{\varepsilon_{o} r}\left(1-e^{-\frac{r^{2}}{a^{2}}}\right) \\
\vec{B}(r)=B_{\theta}(r)=\frac{\rho_{o} a^{2} \beta}{\varepsilon_{o} \gamma^{2}}\left(1-e^{-\frac{r^{2}}{a^{2}}}\right),
\end{gathered}
$$

where $\beta$ and $\gamma$ are the relativistic factors, and $\varepsilon_{0}$ is the permittivity constant $\left(\varepsilon_{\mathrm{o}}=8.8541 \times 10^{-12} \mathrm{Fm}^{-1}\right)$.

The Lorentz force will be radial with a magnitude 


$$
F_{r}(r)=e(\vec{E}+c \vec{\beta} \times \vec{B})=\frac{e \rho_{o} a^{2} \beta}{\varepsilon_{o} \gamma^{2} r}\left(1-e^{-\frac{r^{2}}{a^{2}}}\right) .
$$

For a typical synchrotron this space-charge force is a perturbation to the force of the synchrotron magnets. The equation of motion for a beam particle can be written as

$$
\frac{d^{2} r}{d s^{2}}+[\kappa(s)+k(s)] r=0,
$$

where $\kappa(\mathrm{s})$ is the synchrotron magnetic gradient and $\mathrm{k}(\mathrm{s})$ is the space-charge component given by

$k(s)=\frac{1}{m \gamma \beta^{2} c^{2}} \frac{d F_{r}}{d r}=\frac{e \rho_{o} a^{2}}{\varepsilon_{o} \gamma^{3} \beta^{2} c^{2} m}\left[\frac{2 r}{a^{2}}-\left(\frac{2 r}{a^{2}}-\frac{1}{r^{2}}\right)\left(1-e^{-\frac{r^{2}}{a^{2}}}\right)\right]$.(9)

The largest space-charge perturbation occurs for small amplitude particles (small average $r$ ) where $k(s)$ is independant of $r$. The tuneshift in this region is [6]

$$
\Delta v=\frac{1}{4 \pi} \int_{S} k(s) \beta_{c s}(s) d s,
$$

where $\beta_{c s}$ is the Courant-Snyder amplitude function. Approximating the integral yields

$$
\Delta v_{\max }=\frac{e \rho_{o} S \overline{\beta_{c s}}}{4 \pi \varepsilon_{o} \gamma^{3} \beta^{2} c^{2} m},
$$

where $\overline{\beta_{c s}}$ is the average Courant Snyder amplitude function around the synchrotron. Defining the beam current (I) and the normalized beam emittance $\left(\varepsilon_{\mathrm{n}}\right)$ as $I=\beta c \rho_{o} a^{2} \pi$ and $\varepsilon_{n}=\frac{1}{4} \quad \mathrm{a}^{2} \gamma \beta{\overline{\beta_{c s}}}^{-1}$, the maximum tune shift can be written as

$$
\Delta v_{\max }=\frac{e I S}{16 \pi^{2} \varepsilon_{o} m c^{3} \beta^{2} \gamma^{2} \varepsilon_{n}} .
$$

Larger amplitude particles experience a smaller space charge force and therefore a smaller tuneshift. The general equation for the particle tuneshift as a function of particle amplitude (A) is found to be

$$
\Delta v(A)=\Delta v_{\max } \frac{\left(1-e^{-\eta A \varepsilon_{n}^{-1}}\right)}{\eta A \varepsilon_{n}^{-1}},
$$

where $\eta$ is independent of $A, \varepsilon_{\mathrm{n}}$, and I but is dependant on the synchrotron design and is proportional to $\beta \gamma$ with a proportional constant of order unity. We note that since the beam will have a significant distribution in tune, it is not resonable to assume that the resonant instabilities are determined by the average beam tune, but by the tunes of the individual particles.

\section{METHOD OF INVESTIGATION}

To study the cause of instabilities we have developed a self-consistent computer model[5]. It tracks a set of macro-particles around the synchrotron lattice in full 6-dimensional phase space. For a long, lattice dominated beam with slowly varying radial profile the space-charge force has only a radial component and can be calculated using a two dimensional algorithm. The synchrotron magnets are applied as thin-elements as described by Schachinger and Talman[8]. The tracking method follows.

\subsection{Regions without synchrotron magnets}

Each particle is advanced in space a timestep dt, according to the equations

$$
\begin{aligned}
& x^{n+1}=x^{n}+\beta_{x}^{n} c d t \\
& y^{n+1}=y^{n}+\beta_{y}^{n} c d t \\
& \mathrm{~s}^{\mathrm{n}+1}=s^{n}+\beta_{s}^{n} c d t,
\end{aligned}
$$

where $\mathrm{x}, \mathrm{y}$, and $\mathrm{s}$ are the horizontal, vertical, and longitudinal positions. The space-charge electric field at each particle is calculated using a standard 2-D PIC algorithm and the particle momentums are advanced as

$$
\begin{gathered}
\beta_{x}^{n+1}=\beta_{x}^{n}+\frac{E_{x}}{\gamma^{3}} d t \\
\beta_{y}^{n+1}=\beta_{y}^{n}+\frac{E_{y}}{\gamma^{3}} d t \\
\beta_{s}^{n+1}=\beta_{s}^{n}+\frac{\beta_{s}^{n}}{\gamma^{3}}\left(\beta_{x}^{n} E_{x}+\beta_{y}^{n} E_{y}\right) d t .
\end{gathered}
$$

\subsection{Regions with synchrotron magnets}

When there is a thin element in a particle's path during the time step, the particle is advanced in time, $\mathrm{dt}_{1}$, to the element and there the particle momentum receives its magnetic kick as

$$
\begin{gathered}
\beta_{x}^{+}=\beta_{x}^{-}-\frac{\beta_{s}}{1+\delta} \xi_{y} \\
\beta_{y}^{+}=\beta_{y}^{-}+\frac{\beta_{x}}{1+\delta} \xi_{x} \\
\beta_{s}^{+}=\sqrt{\left(\beta_{s}^{-}\right)^{2}+\left(\beta_{x}^{-}\right)^{2}+\left(\beta_{y}^{-}\right)^{2}-\left(\beta_{x}^{+}\right)^{2}-\left(\beta_{y}^{+}\right)^{2} .}
\end{gathered}
$$

The parameter $\delta$ is the particle's fractional deviation from the design momentum and $\xi$ is calculated from the particle's position and the magnetic poles $\left(a_{n}, b_{n}\right)$ as

$$
\xi_{x}+i \xi_{y}=\sum_{n=0}^{n_{\text {poles }}}\left(b_{n}+a_{n}\right)(x+i y)^{n} .
$$

Using the new momentum the particle is then advanced in space for the remainder of the timestep $\left(\mathrm{dt}_{2}=\mathrm{dt}_{-} \mathrm{dt}_{1}\right)$.

\section{SIMULATION RESULTS}

For our simulations we have used a simple synchrotron lattice comprized of eight bending magnets and eight quadrupole magnets (four focusing and four defocusing). Unless otherwise stated the beam energy was set to $10 \mathrm{MeV}$, beam current 0.5 amps, and beam emittances (horizontal and vertical) $10 \pi \mathrm{mm}$-mrad. The magnetic strengths were adjusted so the horizontal machine tune $\left(v_{\mathrm{xo}}\right)$ was 1.75 , while the vertical machine tune was adjusted to determine regions of instability.

Using the simulation with the vertical machine tune set to 0.85 (away from instabilities), we observed the amplitude dependence of the tune-shift for several 
currents. Fig. 1 compares the computer results with Eq. (6) for currents of $0.1,0.3$, and 0.5 amps.

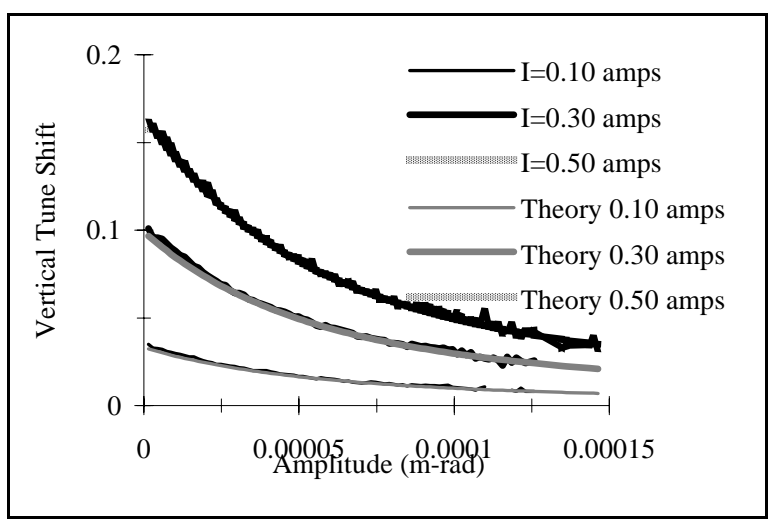

Fig. 1: Comparison of Theoretical Tune and Simulation

The region on integer instability was measured by scanning the vertical machine tune between 1.005 and 1.150. At each tune a simulation run was performed for up to 500 turns and the final saturation emittance was recorded. Fig. 2 shows that the maximum emittance growth is maximum at $v_{\text {yo }}=1.05$ and ranges between 1.03 and 1.13 .

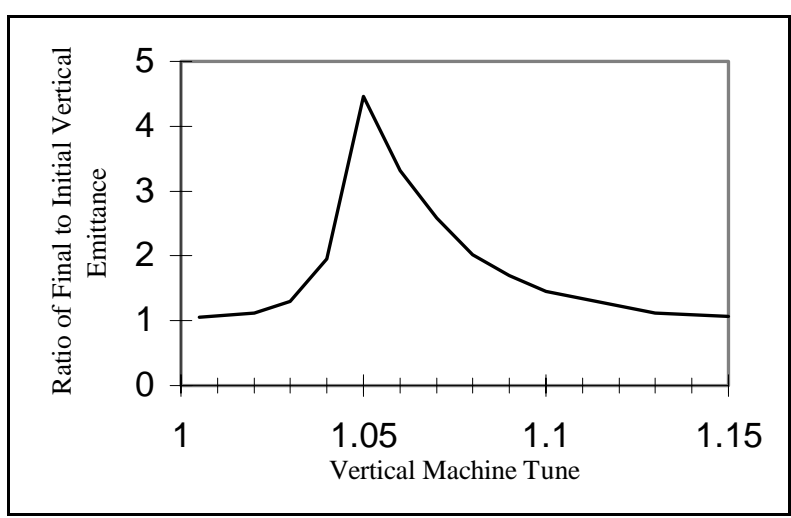

Fig. 2: Amplitude Growth as a function of machine tune

Fig. 3 shows the beam tune $\left(v_{\mathrm{y}}\right)$ as a function of amplitude for five machine tunes $\left(v_{\mathrm{yo}}=1.02,1.05,1.08\right.$, 1.10, and 1.15). Those beams whose tunes cross the integer are unstable, with the largest instability occuring when most of the beam lies slightly below the integer. As the resonant beam particles increase in amplitude the beam density decreases resulting in a lower overall tuneshift. This effect causes particles that had tunes slightly below resonance to become resonant. Typically the beam will be unstable when particles with amplitudes 1-20 times that of the beam emittance have integral tunes. The upper bound is set to be 20 by the scarcity of particles at such large amplitudes and the lower bound by the steep slope of the tune distribution at low amplitudes. The unstable band of machine tune in the vicinity of the integer resonance of $n$ is

$$
n+\frac{\Delta v_{\max }}{20 \eta}\left(1-e^{-20 \eta}\right)<v_{o}<n+\frac{\Delta v_{\max }}{\eta}\left(1-e^{-\eta}\right) .
$$

For our parameters, with $\eta=0.32, \Delta v_{\max }=0.165$, and $\mathrm{n}=1$, the range of instability is $1.025<v_{o}<1.13$.

This agrees with the region of instability from our simulation shown in Fig. (2).

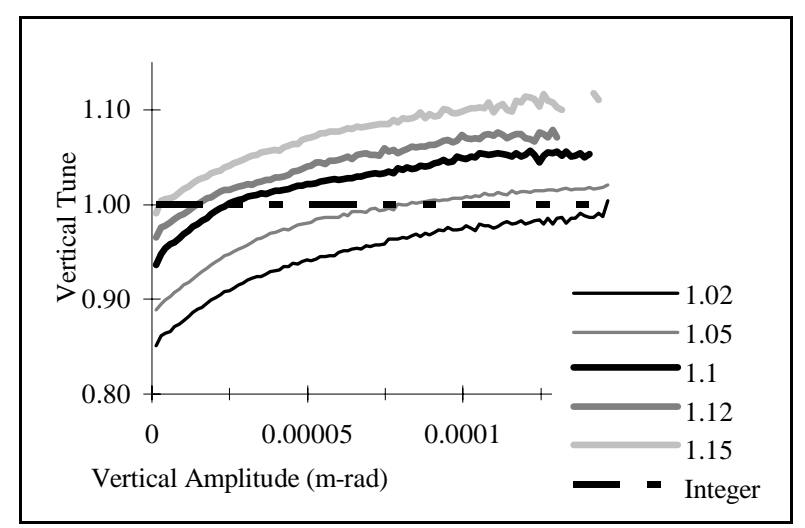

Fig. 3: Tune distribution as it crosses resonance

\section{CONCLUSIONS}

To understand the region of integral resonance, it is necessary to include the tune spread as a function of amplitude. A Gaussian beam will be unstable when particles with an amplitude greater than the rms emittance have integral tune. The resonance has the effect of increasing the beam emittance (which decreases the tune shift) until all of the individual particle tunes are above the resonant tune. The emittance growth is maximum when most, but not all, beam particles lie below the resonant tune. When all of the particles lie below the resonant tune, the beam is stable. Work supported by DOE and Hitachi.

\section{REFERENCES}

[1] A.W. Chao. Physics of Collective Beam Instabilities in High Energy Accelerators (John Wiley \& Sons, New York, 1993).

[2] R. Baartman. Proceedings of the International Workshop on Particle Dynamics in Accelerators, edited by S. Machida and K. Hirata (1995) p. 273.

[3] S. Machida. Nucl. Inst. \& Methods. A309, 43 (1991)

[4] W.T. Weng. AIP Conference Proceedings 153, 349 (1987).

[5] M.B. Ottinger, T. Tajima, and K. Hiramoto. Submitted to J. Comp. Phys., November 1996

[6] E.D. Courant and H.S. Snyder. Annals of Physics, 3, 1 (1958).

[7] R.C. Davidson. Physics of Nonneutral Plasmas (Addison-Wesley Publishing, Redwood City, California, 1990).

[8] L. Schachinger and R. Talman. Particle Accelerator 22, 35 (1987). 